\title{
Effects of Acupuncture Treatment on Postoperative Gastrointestinal Dysfunction in Colorectal Cancer: Study Protocol for Randomized Controlled Trials
}

\section{Xueyan Liu ( $\nabla$ xueyan580@163.com )}

Beijing University of Chinese Medicine https://orcid.org/0000-0003-2921-2667

\section{Zhijie Wang}

Cancer Hospital Chinese Academy of Medical Sciences

\section{Hao Yao}

Beijing University of Chinese Medicine

\section{Yanrong Yang}

Beijing University of Chinese Medicine

\section{Huijuan Cao}

Beijing University of Chinese Medicine

\section{Zhanhao TOH}

singa care medical

\section{Yi Ren}

Beijing University of Chinese Medicine Affiliated Dongzhimen Hospital

\section{Ruwen Zheng}

bei jing zhong yi yao da xue dong zhi men yi yuan: Dongfang Hospital

\section{Research Article}

Keywords: Colorectal cancer, Postoperative, Acupuncture, Gastrointestinal function, Enhanced Recovery After Surgery

Posted Date: July 6th, 2021

DOl: https://doi.org/10.21203/rs.3.rs-294977/v1

License: (c) (i) This work is licensed under a Creative Commons Attribution 4.0 International License. Read Full License

Version of Record: A version of this preprint was published at Trials on January 31st, 2022. See the published version at https://doi.org/10.1186/s13063-022-06003-7. 


\section{Abstract}

Background: Postoperative Gastrointestinal Dysfunction (PGID) is a common complication arising from colorectal cancer surgery. Attributing factors such as anesthesia, surgical retraction, early intake of water, etc. can inhibit gastrointestinal motility, causing constipation, reduction or absence of bowels sounds, nausea, vomiting and other symptoms. Delayed recovery in gastrointestinal function can lead to intestinal obstructions or paralysis, anastomotic leaks and other complications, affecting the patient's recovery and quality of life negatively. Due to its complex pathophysiology, treatment for PGID in colorectal patients has remained a challenge. Acupuncture is an alternative therapy commonly used for post-operative recovery. This study aims to evaluate the therapeutic efficacy and safety of Acupuncture on PGID. Through the complementation of Acupuncture and Enhanced Recovery After Surgery (ERAS) protocols, the advantages of acupuncture treatments could be demonstrated to promote its application in future clinical practice.

Methods/design: The study design is a prospective randomized controlled trial (RCT). 160 postoperative colorectal cancer patients will be recruited from Cancer Hospital Chinese Academy of Medical Sciences (CICAMS). Subjects who fulfill inclusion criteria will be randomly assigned into Acupuncture group (AG) $(n=80)$ or control group $(C G)(n=80)$. AG will receive Acupuncture treatment and ERAS care protocols, CG will only receive ERAS care protocols. Intervention will begin on the first day post-surgery, continuing for 4 days, with a follow-up assessment in a month. Time of first postoperative flatus would be the primary outcome measure. Secondary outcome measures include time of first postoperative defecation, time of first fluid intake, time of first ambulation, postoperative hospital stay, gastrointestinal reaction score, postoperative quality of life, acupuncture sensation evaluation scale. All results are evaluated from baseline, post-treatment and upon follow-up.

Discussion: The results of the study would help elucidate evidence of the therapeutic effects of acupuncture on recovery of postoperative gastrointestinal function. The objective of the study aims for the eventual inclusion of acupuncture in the ERAS protocol, allowing for wider application in clinical practice.

Trial Registration: Clinicaltrials.gov: ChiCTR2000036351. Register August 22,2020.

\section{Background}

Colorectal Cancer is a common malignant condition involving the gastrointestinal tract. With ageing population and changes in diet structures, incidence and mortality rates of colorectal cancer have seen a rising trend through the years [1]. Epidemiological studies have shown[2,3] that in 2016, newly diagnosed cases and deaths of colorectal cancer in United States were 135000 and 50000 respectively, and China has a higher ratio of newly diagnosed cases to death(50.8\%) when compared with the United States(37\%). In China, incidence of colorectal cancer in male and females ranked 4th and 3rd respectively, while mortality rates ranked 5 th and 4 th respectively. The incidence of colorectal cancer for 
the age group below 50 has also shown an increasing trend. Surgical intervention remains the main treatment for colorectal cancer, despite surgical trauma and postoperative complications. Postoperative gastrointestinal dysfunction (PGID) is one of the most common complications, and is often associated with administer of anesthesia, surgical retraction, postoperative fasting, usage of analgesics, prolong bed rest etc.[4,5], and include symptoms such as distention, absence of flatus or bowel, accompanied by varying degrees of nausea, vomiting and fever, leading to longer recovery periods, decrease in quality of life and increase consumption of medical resources[6]. Current methods to promote recovery of gastrointestinal function include postoperative conventional treatments and prokinetic drugs such as domperidone and cisapride. However, the therapeutic efficacy of these drugs are less than ideal and accompanied with various side effects, possibly due to over-stimulation of gastrointestinal motility that could affect postoperative recovery negatively[7].

Enhanced Recovery After Surgery (ERAS) is an evidence-based approach that employs a series of multimodal and optimized peri-operation measures to reduce surgical trauma and stress response, promote physiological and psychological well-being, and facilitate faster recovery [8]. As a newly implemented peri-operative management principle, specific measures include: preoperative education and counseling, selective preoperative bowel preparation, selective preoperative sedation, 30-60min preoperative antibiotic prophylaxis, standardized anesthetic protocol, perioperative fluid intake management, prevention of intraoperative hypothermal, early removal of urinary catheters, multimodal postoperative analgesia, early postoperative food intake, early mobilization etc.[9]. ERAS protocol has the advantages of faster recovery, lesser complications, shorter hospitalization and lower medical cost, and is widely adopted and implemented in various surgical fields [10]. With rapid developments in the field of surgical rehabilitation, measures to improve postoperative recovery in colorectal cancer patients are gradually being optimized. At present, although ERAS has lowered incidence of postoperative complications, a portion of patients, in particular elderly, diabetic patients, or individuals who have poor digestive functions, could still develop long-term, refractory, recurrent complications [11].

Acupuncture is a unique and natural treatment method originating from China, and practice of which to treat digestive ailments had been recorded over a few thousand years [12]. Recent evidence shows that $[13,14]$ acupuncture exhibits higher effectiveness over western medications, displaying various advantages such as expediting recovery of gastrointestinal function, reducing postoperative complications, boosting immunity and improving quality of life, when applied as a complementary treatment for colorectal cancer. Additionally, acupuncture possesses qualities of a safe and economical treatment, and holds an irreplaceable role in rehabilitation medicine [15]. However, existing studies have their limitations, including small study samples, poorly designed experiments, low credibility of experimental results etc. Our study aims to further investigate the therapeutic efficacy of acupuncture on postoperative recovery of gastrointestinal functions, by comparing trial results through complementation of acupuncture and ERAS protocols-guided laparoscopic radical resection of colon cancer versus solely ERAS protocols-guided laparoscopic radical resection of colon cancer, to provide statistical evidence for the inclusion of acupuncture in the ERAS protocols for colorectal cancer. 


\section{Methods And Design}

Aim

1. To determine therapeutic efficacy of acupuncture on the recovery of postoperative gastrointestinal function in colorectal cancer patients.

2. To provide statistical evidence for the inclusion of acupuncture into ERAS protocols.

\section{Study Design}

The study is a single center, prospective, randomized controlled clinical trial. Recruitment for study subjects began from 16 October 2020, and is estimated to end by June 2021. 160 patients who are scheduled to undergo laparoscopic radical resection for colorectal cancer will be recruited from the Cancer Hospital Chinese Academy of Medical Sciences (CICAMS). Subjects who fulfill inclusion criteria and signed the informed consent form will be randomly assigned to either the Acupuncture group (AG) or Control group (CG) in a 1:1 ratio. CG will receive only ERAS care protocols. AG will receive both acupuncture and ERAS care protocols, with acupuncture sessions conducted once per day over 4 days, beginning on the first day after surgery, with each session lasting 30mins. Assessments are made prior to treatments, and on the $4^{\text {th }}$ day and 1 month after the treatments. Fig. 1 and Fig. 2 show the timeline of enrolment, intervention, evaluation, and follow-up assessments.

\section{Randomization and Allocation Concealment}

Patients who fulfill inclusion criteria will be randomly allocated to AG or CG in a 1;1 ratio. Randomized allocation sequence will be generated using Statistical Analysis System, SAS 9.3 (SAS Institute Inc. Cary, NC, USA), by an appointed investigator who is not involved in the recruitment or evaluation process. The generated sequences will be placed in serialized, opaque, sealed envelopes (to ensure concealment of allocation from the evaluators). After ensuring patients fulfill inclusion criteria and informed consent forms are obtained from them, the envelopes are opened by the acupuncturist and acupuncture treatment is carried out (the acupuncturist is excluded from assessment and statistical analytic process as blinding of the acupuncturist cannot be achieved).

\section{Blinding Method}

Throughout the study, the statisticians and evaluators are blinded, so that independent assessments and statistical analysis can be carried out. The subjects in the control group could not be blinded due to their particularity. Acupuncturist were not blinded in this study. Unblinding will only be allowed under emergencies such as incidence of serious adverse effects.

\section{Study Enrolment and Recruitment}

Patients are recruited from the Colorectal Surgical In-patient Department of CICMAS through publication and distribution of posters and leaflets containing information of the study. 


\section{Inclusion Criteria}

To enroll in the study, subjects must: 1) be diagnosed with colorectal cancer based on cytological or histopathological features 2 ) be scheduled to undergo laparoscopic radical resection for colorectal cancer 3) age between 18-80 years old 4) consent to participation and completion of the full treatment process, and be able to sign the informed consent form.

\section{Exclusion Criteria}

Patients may not participate in the study if they: 1) refuse to undergo laparoscopy treatment 2) have acute intestinal obstruction or intestinal perforation 3) have a history of other malignant neoplasms, or are having other malignant tumors concurrently, 4) have undergone vagotomy or colostomy 5) have preexisting conditions such as severe cardiovascular disease, hepatic, renal, hematopoietic, immune system conditions, psychiatric disorders, hyper- or hypothyroidism, diabetes, spinal cord injury 6) have skin allergies or infections 7) are anxious or fearful of acupuncture treatments 8) have been prescribed adrenergic agonist or antagonist, cholinergic or anticholinergic medications that affect gastrointestinal function for the past 1 month 9) have severe postoperative complications such as anastomotic leak, intra-abdominal infection 10) are pregnant or lactating.

\section{Intervention}

Study subjects from both groups will receive standardized peri-operative care from ERAS protocols (Detailed nursing and care plans will be facilitated by the CICAMS, and would not be intervened by investigators).

\section{Acupuncture group}

Patients in AG will receive acupuncture treatment in addition to ERAS care protocols. Acupuncture treatment will be performed once daily, consecutively over 4 days, starting on the first day after the laparoscopy. Patients will assume supine position for the treatment. Treatment will be carried out by a licensed acupuncturist who holds a China Acupuncturist Certification, with at least 2 years of clinical experience in acupuncture. Site of insertions will be swabbed and disinfected with $75 \%$ alcohol prior to needle insertion. Sterile, single-use acupuncture needles with diameter $0.30 \mathrm{~mm}$ and length $40 \mathrm{~mm}$ (China Suzhou, Huatuo) are used. Acupuncture points are selected based on Traditional Chinese Medicine (TCM) theory and clinical experience: ST36 Zusanli, ST37 Shangjuxu, PC6 Neiguan, L14 Hegu (Fig. 3). Exact locations and depth of insertions are referenced from the 2006 National Standards of the People's Republic of China (GB/T12346-2006) "Nomenclature and Location of Acupuncture Points"[16] (Fig. 4). After insertion of needles, stimulation of acupoints will be performed every 10 minutes, through liftingthrusting and twirling-twisting manipulations, to achieve sensations of deqi. Needles are kept inserted for 30mins.

Figure 4 Locations and manipulations of acupuncture group and control group 


\section{Control group}

Patients in CG will only receive ERAS care with no acupuncture intervention.

\section{Measures of Outcomes}

\section{Primary Outcome}

Time of first postoperative flatus, is the study's primary outcome, and is measured as the time period starting from the patient's return to ward after the laparoscopy procedure till the first passage of flatus. It is obtained through the assistance of family members and caregivers, via inquiry and record of patient's status.

\section{Secondary Outcomes}

Secondary outcomes include time of first postoperative defecation, time of first fluid intake, time of first ambulation, which are obtained through assistance of family members and caregivers, via inquiry and records of patient's status. Other secondary outcome measures such as gastrointestinal reaction score (postoperative pains, distention, nausea, vomiting), postoperative hospital stay, quality of life, acupuncture sensation evaluation scale, as well as other postoperative complications (fever, pneumonia, wound infection, bleeding etc.) are assessed and evaluated by our investigators.

Postoperative pain: Measured postoperatively from day 1 to 4 at 9.00 in the morning, using Visual Analog Scores (VAS), and tabulated with dosage and frequency of Patient Controlled Analgesia (PCA) and analgesic medication used.

Gastrointestinal reaction score: Extent of distention is recorded using the Likert scale [17], postoperatively from day 1 to 4 at 9.00 in the morning. Extent of nausea and frequency of vomit is recorded using the VAS, postoperative from day 1 to 4 , at 9.00 in the morning.

Postoperative hospital stay: Measured from the day of the laparoscopy procedure till day of discharge. Discharge criteria includes: 1) Controllable pains without use of analgesics 2) Absence of nausea or vomiting 3) Normal passing of flatus or bowels 4) Tolerance towards soft diets 5) Ability to ambulate independently or self-care.

Postoperative complications: Complications are recorded and evaluated using the Clavien Dindo Classification [18].

Acupuncture Sensation Evaluation Scale: Acupuncture sensations are assessed after every acupuncture treatment session through inquiring about patient's sensations. Venous blood will also be collected on the day prior to operation, as well as the first and third day after the laparoscopy, to assess safety of acupuncture base on blood examinations and biochemical indicators. 
Quality of life: Assessed through Quality of Life Questionnaire (QLQ-c30), provided to patients prior to operation and 1 month after the operation [19].

\section{Safety Assessments}

All adverse events should be reported by patients proactively, directly observed by clinical physicians, or monitored closely by asking patients of adverse reaction-related incidences. Detailed records of adverse events should be documented in the case report forms (CRFs), and appropriate treatment provided where necessary. If serious adverse events occur, the relevant intervention will be terminated and investigators are to report to the Research Ethics Committee immediately.

\section{Sample Size}

Our study estimates that a mean decrease of 8hrs in time of first flatus after application of acupuncture to promote recovery in postoperative gastrointestinal functions is of significant value. Literature review showed standard deviation of time of first flatus between study group and control group to be 12.28 and 14.57 respectively. Using a unilateral test of superiority, PASS.11 software is used to compute sample size. Using $a=0.05, \beta=0.1$, Power $=0.9,68$ subjects are required in each group, with a total of 136 subjects needed for the study. Estimating a $20 \%$ dropout rate, a minimum recruitment of 160 subjects is required.

\section{Statistical Analysis}

Guidelines are provided by statistician to the principal investigator, to formulate plans for statistical analysis and database setup, and SPSS software will be used to analyze the collected data. The entire data will be processed by the statistician. Statistical analysis will be carried out using SPSS 22.0, using the Intention-to-Treat (ITT) principle. A one-sided test is applied to the data, with $p$-value $<0.05$ considered significant. For descriptive analysis, qualitative variables are described by frequency tables and percentages, quantitative variables are described by mean values and standard deviations. For comparison between two groups, we will use T-test if quantitative variables exhibits normal distribution, homogeneity of variance test will be conducted between groups using $p$ value of 0.05 . If variance is unequal, Welch-Satterthwaite T-test is used. Wilcoxon rank-sum test is used if data do not follow normal distribution. Statistical analyses of primary therapeutic efficacy indicators are confirmed by the Statistics department of Bejing University of Chinese Medicine.

\section{Data Collection and Monitoring}

Data collection will be carried out by an attending surgeon. Studied variables are assessed daily from day 1 to day 4 after the laparoscopy procedure, and recorded on the CRFs. A month after the operation, a follow-up would be carried out through phone calls, to gather responses for the Quality of Life Questionnaire (QLQ-c30). Data collected will include actual number of participants, excluded case studies, demographics and other baseline variables, compliance rates, safety assessments, incidence of complications from the operation, general treatment outcomes, and therapeutic efficacy. Adverse events will also be documented. Data will be collected over the entire study duration, and managed confidentially 
and securely. Throughout the study, unless otherwise indicated, study subjects will be addressed using designated serial numbers and not their names. Data will only be accessible to investigators who have signed the confidential disclosure agreement, the organization involved in the study, as well as government auditors. The Research Ethics Committee of Dong Fang Hospital will exercise supervision over the collection and management of patients' data.

\section{Quality Control}

To ensure quality control of the study, our methodologists, acupuncturists, surgeons and anesthetists will discuss and review research protocols continuously. Prior to the start of the study, all research personnel will have to undergo training to familiarize with research plans, experimental timelines, standardized treatment protocols etc. A quality controller appointed by the principal investigator will review CRFs fortnightly. Recorded data on CRFs are not permitted to edits. If any changes are made, a detailed explanation and signature by the modifier must be provided. A meeting will be held every three months to report and provide updates on the progress of the study.

\section{Ethical Issues}

The study has been developed in accordance with the Declaration of Helsinki and principles from the Good Clinical Practice Guide.

\section{Ethics Approval}

The study has been approved by the Research Ethics Committee of Dong Fang Hospital, Beijing University of Chinese Medicine. The trial (ChiCTR2000036351) is registered on the Chinese Clinical Trial Registry (CHiCTR) platform on 22 August 2020.

\section{Consent and Confidentiality}

Informed consent forms will be obtained from participating subjects prior to enrolment. Collected data are kept in confidentiality through the entire study.

\section{Discussions}

Gastrointestinal dysfunction is a major factor that affects postoperative recovery in colorectal cancer patients and could lead to poor prognosis. Therefore, prevention of PGID in colorectal cancer patient to reduce peri-operative complications and hospitalization cost is of important significance [20]. Current western medical interventions such as gastrointestinal decompression and oral administered prokinetic medications have shown unsatisfactory results in patients, leading to the search of alternative treatment options by clinical practitioners, to facilitate early recovery of gastrointestinal functions [7]. Acupuncture is a safe and effective treatment method that could promote gastrointestinal functions while having minimal side effects $[13,15]$. Although current studies have shown duration of PGID could be reduced through acupuncture, research on acupuncture and its role and therapeutic effect in prevention of PGID in 
colorectal cancer patients has not been studied. With recent developments in enhanced recovery principles, improving postoperative quality of life in patients has gained importance [22]. Therefore, our study aims to determine the effects of acupuncture intervention protocols on the recovery of postoperative gastrointestinal functions in colorectal cancer patient.

Postoperative gastrointestinal dysfunction in colorectal cancer patient is recognized as "Chang $\mathrm{Bi}$ " or "Chang Jie" in Traditional Chinese Medicine theory. Based on TCM organ system theory, acupoints are mostly selected from the spleen and stomach meridian to promote recovery of gastrointestinal functions. Acupoints include LI 4, PC6, ST36, ST37. ST36 improves blood flow and distribution within the gastrointestinal tract, regulates motilin production through somatostatin, and improves peristalsis through regulation of NO which increases production of angiotensin [23,24]. ST37 is a lower confluent acupoint for the large intestines, and is often used in conjunction with ST36 to treat large intestine-related conditions such as ulcerative colitis, irritable bowel syndrome [25]. PC6 regulates endocrine functions, in particular epinephrine and vasopressin, to reduce stomach acid secretions and regulate gastrointestinal functions [26]. PC6 is also frequently used in the prevention of postoperative nausea caused by the use of opioids and other medications [27]. LI4 is the yuan-primary point of the large intestine meridian, and is often used in the treatment of large intestine-related conditions, and for its analgesic properties [28].

Patients between ages 18-80 have been included in the study to cover a larger spectrum of the demographics. At the same time, assessment of gastrointestinal function related clinical parameters and serological tests is done to aid in the evaluation of the efficacy and safety of acupuncture treatments, ultimately providing the basis for the overall evaluation of the effects of acupuncture on recovery of gastrointestinal function from colorectal cancer operations. Clinical parameters for gastrointestinal function include time of first postoperative flatus, time of first postoperative defecation, time of first fluid intake, hospitalization period etc. Importantly, serological tests such as blood test and biochemical indicators have been included in our study to assess the safety of acupuncture. One of the advantages of our study is the conduct of a follow-up to gather response through the QLQ-c30 to assess patients' quality of life one month after the operation, which will reflect the effects of acupuncture on quality of life of postoperative colorectal patients.

However, there are limitations to the study. Firstly, ensuring punctual and accurate follow-ups is both an important and difficult task. Secondly, the study lacks a sham acupuncture control group, due to difficulties in blinding of study subjects. Lastly, insertion of rectal tubes in a portion of our study subjects might cause inaccuracies in reporting time of first postoperative flatus by the patients due to unconscious passing of flatus via rectal tubes. Despite these limitations, the study should elucidate the general effect of acupuncture on postoperative recovery.

In conclusion, we hope that through rigorous clinical study, statistical evidence of acupuncture in promoting recovery of gastrointestinal function in post-laparoscopy colorectal cancer patients can be demonstrated, providing the basis for the inclusion of acupuncture into ERAS principles and protocols, to 
encourage the application of acupuncture in the aforementioned condition after conventional surgical procedures.

\section{Declarations}

\section{Acknowledgements}

Firstly, the author would like to express gratitude towards Dr Liu Qian, Cancer Hospital Chinese Academy of Medical Sciences, for his support and guidance towards the study. I would also like to thank Cao Hui Juan, Yao Hao and Yang Yan Rong from Dong Fang Hospital, Beijing University of Chinese Medicine, for their support in statistical analysis. I am also grateful to Dr Wang Zhi Jie, Cancer Hospital Chinese Academy of Medical Sciences, for his participation as an attending surgeon in our study. Lastly, I would like to acknowledge the support provided by the entire team from Department of Colorectal Surgery, Cancer Hospital Chinese Academy of Medical Sciences.

\section{Authors' contributions}

$\mathrm{XyL}, \mathrm{HY}, \mathrm{YrY}$ and RwZ all contributed to the design and development of the study protocol. $\mathrm{XyL}$ is responsible for the performing of this study and the writing of this manuscript; $\mathrm{YrY}$ is responsible for the generation of random numbers, $\mathrm{HY}$ is responsible for the statistics and analysis of data. $\mathrm{HjC}$ gave guidance to statistical methodology. ZjW is a doctor in charge of the collaborative hospital, and is responsible for patient recruitment and data collection. ZHT translated and checked the manuscript. RwZ and $\mathrm{YR}$ is the general supervisor for this research. All authors read and approved the final manuscript.

\section{Funding}

This study is supported by the Outstanding Teacher Educational Research of Dong fang Hospital of Beijing University of traditional Chinese medicine, the 1166 Talent Training Program of Dong Fang Hospital, Beijing University of traditional Chinese Medicine》and the Scientific Research Fund Project of Beijing University of traditional Chinese medicine.

\section{Availability of data and materials}

The datasets used and/or analyzed during the current study are available from the corresponding author on reasonable request.

\section{Ethics approval and consent to participate}

This study protocol and informed consent materials have been approved by the Ethics Committee of Dong Fang Hospital, Beijing University of TCM; Committee approval number: JDF-IRB-2019036002. Written, informed consent to participate will be obtained from all participants.

\section{Consent for publication}


Not applicable.

\section{Conflicting Interests}

The authors declare they have no conflicting interests.

\section{Study Status}

Recruitment began on 16 October 2020. Recruitment is anticipated to end on September 2021. Recruitment for the study is currently ongoing.

\section{References}

1. Dehal AN, Graff-Baker AN, Vuong B, et al. Correlation Between Clinical and Pathologic Staging in Colon Cancer: Implications for Neoadjuvant Treatment.J Gastrointest Surg,2018,22(10) : 17641771.

2. Chen W, Zheng R, Baade PD, Zhang S, Zeng H, Bray F, Jemal A, Yu XQ, He J. Cancer statistics in China, 2015. CA Cancer J Clin. 2016 Mar-Apr;66(2):115-32.

3. Nfonsam VN, Jecius H, Chen D, Omesiete PN, Ewongwo AN, Elquza E, Scott AJ, Jandova J. Increasing Incidence of Colon Cancer in the Young: Assessing the Tumor Biology. J Am Coll Surg. 2019 Jul;229(1):79-90.

4. Furnes B, Storli KE, Forsmo HM, Karliczek A, Eide GE, Pfeffer F. Risk Factors for Complications following Introduction of Radical Surgery for Colon Cancer: A Consecutive Patient Series. Scand J Surg. 2019 Jun;108(2):144-151.

5. Hida K, Yamaguchi T, Hata H, Kuroyanagi H, Nagayama S, Tada H, Teramukai S, Fukushima M, Koizumi K, Sakai Y. Risk factors for complications after laparoscopic surgery in colorectal cancer patients: experience of 401 cases at a single institution. World J Surg. 2009 Aug;33(8):1733-40.

6. Pędziwiatr M, Mavrikis J, Witowski J, Adamos A, Major P, Nowakowski M, Budzyński A. Current status of enhanced recovery after surgery (ERAS) protocol in gastrointestinal surgery. Med Oncol. 2018 May 9;35(6):95.

7. Cao LX, Chen ZQ, Jiang Z, Chen QC, Fan XH, Xia SJ, Lin JX, Gan HC, Wang T, Huang YX. Rapid rehabilitation technique with integrated traditional Chinese and Western medicine promotes postoperative gastrointestinal function recovery. World J Gastroenterol. 2020 Jun 21;26(23):32713282.

8. Gustafsson UO, Scott MJ, Hubner M, Nygren J, Demartines N, Francis N, Rockall TA, Young-Fadok TM, Hill AG, Soop M, de Boer HD, Urman RD, Chang GJ, Fichera A, Kessler H, Grass F, Whang EE, Fawcett WJ, Carli F, Lobo DN, Rollins KE, Balfour A, Baldini G, Riedel B, Ljungqvist O. Guidelines for Perioperative Care in Elective Colorectal Surgery: Enhanced Recovery After Surgery (ERAS ${ }^{\circledR}$ ) Society Recommendations: 2018. World J Surg. 2019 Mar;43(3):659-695.

9. Nygren J, Thacker J, Carli F, Fearon KC, Norderval S, Lobo DN, Ljungqvist O, Soop M, Ramirez J; Enhanced Recovery After Surgery Society. Guidelines for perioperative care in elective rectal/pelvic 
surgery: Enhanced Recovery After Surgery (ERAS $\left.{ }^{\circledR}\right)$ Society recommendations. Clin Nutr. 2012 Dec;31(6):801-16.

10. Varadhan KK, Neal KR, Dejong $\mathrm{CH}$, Fearon KC, Ljungqvist O, Lobo DN. The enhanced recovery after surgery (ERAS) pathway for patients undergoing major elective open colorectal surgery: a metaanalysis of randomized controlled trials. Clin Nutr. 2010 Aug;29(4):434-40.

11. Bagnall NM, Malietzis G, Kennedy RH, Athanasiou T, Faiz O, Darzi A. A systematic review of enhanced recovery care after colorectal surgery in elderly patients. Colorectal Dis. 2014 Dec;16(12):947-56.

12. Li H, He T, Xu Q, Li Z, Liu Y, Li F, Yang BF, Liu CZ. Acupuncture and regulation of gastrointestinal function. World J Gastroenterol. 2015 Jul 21;21(27):8304-13.

13. Kim KH, Kim DH, Kim HY, Son GM. Acupuncture for recovery after surgery in patients undergoing colorectal cancer resection: a systematic review and meta-analysis. Acupunct Med. 2016 Aug;34(4):248-56.

14. Liu Y, May BH, Zhang AL, Guo X, Lu C, Xue CC, Zhang H. Acupuncture and Related Therapies for Treatment of Postoperative lleus in Colorectal Cancer: A Systematic Review and Meta-Analysis of Randomized Controlled Trials. Evid Based Complement Alternat Med. 2018 Jul 29;2018:3178472.

15. Xin C, Sun JH. The value of acupuncture-moxibustion in enhance recovery after surgery. Zhongguo Zhen Jiu. 2020 Jun 12;40(6):679-82. Chinese.

16. General Administration of Quality Supervision, Inspection and Quarantine of the People's Republic of China, Standardization Administration of the People's Republic of China. Nomenclature and location of acupuncture points. GB/T 12346-2006.

17. Jamieson S. Likert scales: how to (ab)use them. Med Educ. 2004; 38(12): 1212-1218.

18. Dindo D, Demartines N, Clavien P-A. Classification of surgical complications: a new proposal with evaluation in a cohort of 6336 patients and results of a survey. Ann Surg 2004;240:205-13.

19. Aaronson NK, Ahmedzai S, Bergman B, Bullinger M, Cull A, Duez NJ, Filiberti A, Flechtner H, Fleishman SB, de Haes JC, et al. The European Organization for Research and Treatment of Cancer QLQ-C30: a quality-of-life instrument for use in international clinical trials in oncology. J Natl Cancer Inst. 1993 Mar 3;85(5):365-76.

20. Pei KY, Asuzu DT, Davis KA. Laparoscopic colectomy reduces complications and hospital length of stay in colon cancer patients with liver disease and ascites. Surg Endosc. 2018 Mar;32(3):12861292.

21. Ng SS, Leung WW, Mak TW, Hon SS, Li JC, Wong CY, Tsoi KK, Lee JF. Electroacupuncture reduces duration of postoperative ileus after laparoscopic surgery for colorectal cancer. Gastroenterology. 2013 Feb;144(2):307-313.e1.

22. Ljungqvist O, Scott M, Fearon KC. Enhanced Recovery After Surgery: A Review. JAMA Surg. 2017 Mar 1;152(3):292-298.

23. Lu MJ, Yu Z, He Y, Yin Y, Xu B. Electroacupuncture at ST36 modulates gastric motility via vagovagal and sympathetic reflexes in rats. World J Gastroenterol. 2019 May 21;25(19):2315-2326. 
24. Lee CH, Kim DK, Yook TH, Sasaki M, Kitamura N. Effectiveness of electroacupuncture at Zusanli (ST36) on the immunohistochemical density of enteroendocrine cells related to gastrointestinal function. J Acupunct Meridian Stud. 2012 Apr;5(2):63-71.

25. Zhao JM, Li L, Chen L, Shi Y, Li YW, Shang HX, Wu LY, Weng ZJ, Bao CH, Wu HG. Comparison of the analgesic effects between electro-acupuncture and moxibustion with visceral hypersensitivity rats in irritable bowel syndrome. World J Gastroenterol. 2017 Apr 28;23(16):2928-2939.

26. Murakami H, Li S, Foreman R, Yin J, Hirai T, Chen JDZ. Ameliorating Effects of Electroacupuncture on Dysmotility, Inflammation, and Pain Mediated via the Autonomic Mechanism in a Rat Model of Postoperative lleus. J Neurogastroenterol Motil. 2019 Apr 30;25(2):286-299.

27. Yang J, Jiang Y, Chen Y, Sun M, Chen J, Zheng Q, Liang FR. Acupressure the PC6 point for alleviating postoperative nausea and vomiting: A systematic review protocol. Medicine (Baltimore). 2019 Aug;98(33):e16857.

28. Ao L, Shi J, Bai Y, Zhang S, Gan J. Effects of transcutaneous electrical acupoint stimulation on perioperative immune function and postoperative analgesia in patients undergoing radical mastectomy: A randomized controlled trial. Exp Ther Med. 2021 Mar;21(3):184.

\section{Figures}




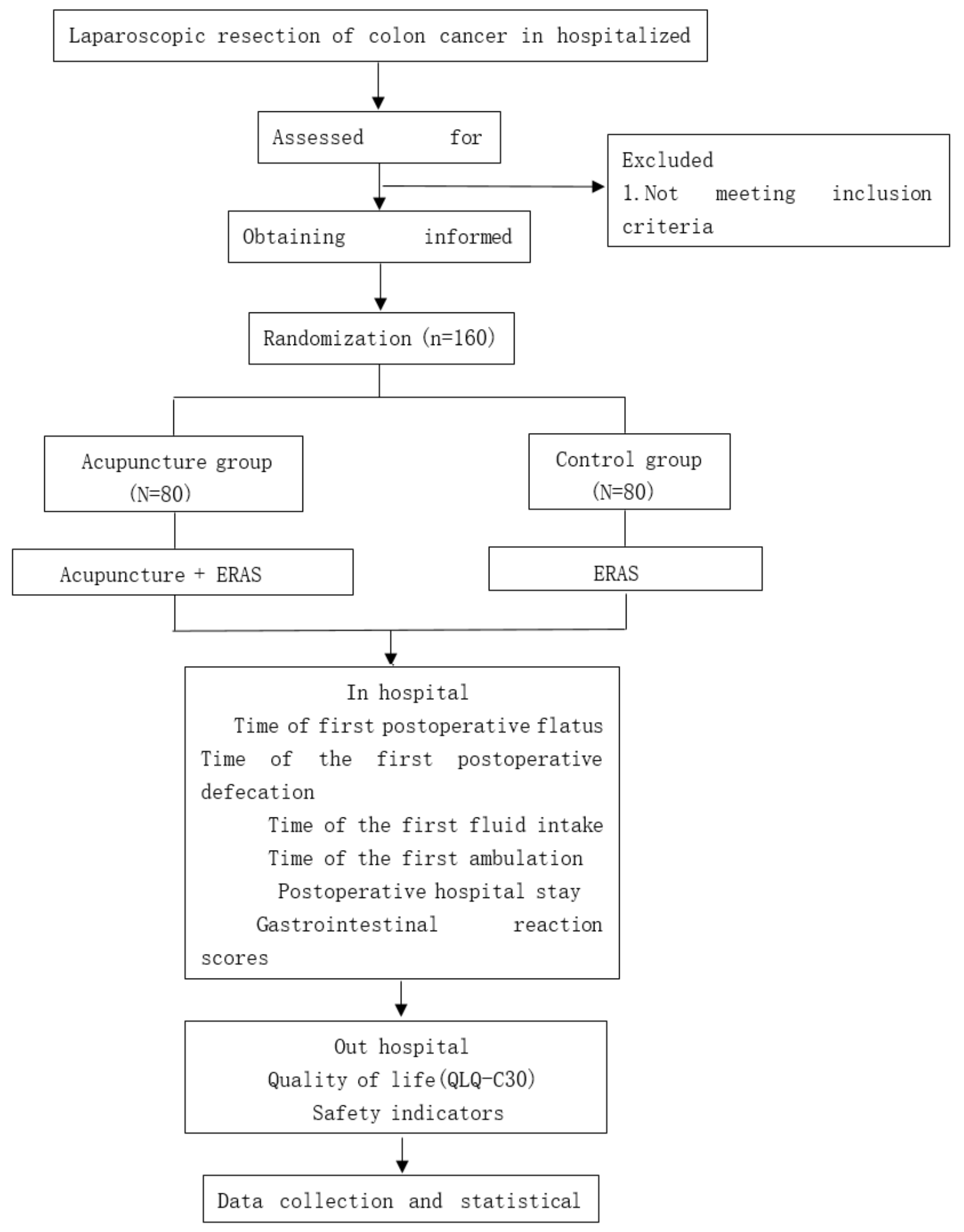

\section{Figure 1}

Flowchart of study design. 


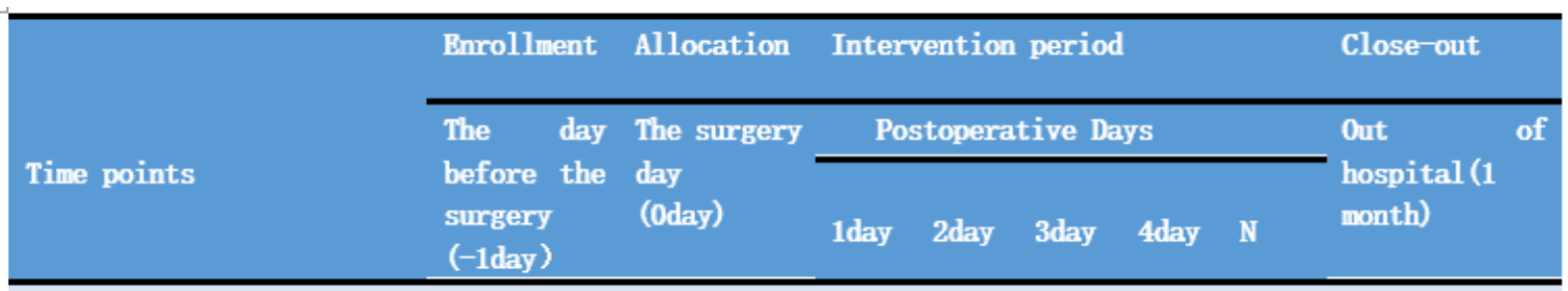

Enrolment

Eligibility screen $\diamond$

Informed consent $\quad$
Allocation
$\diamond$

Interventions

Acupuncture group

Control group

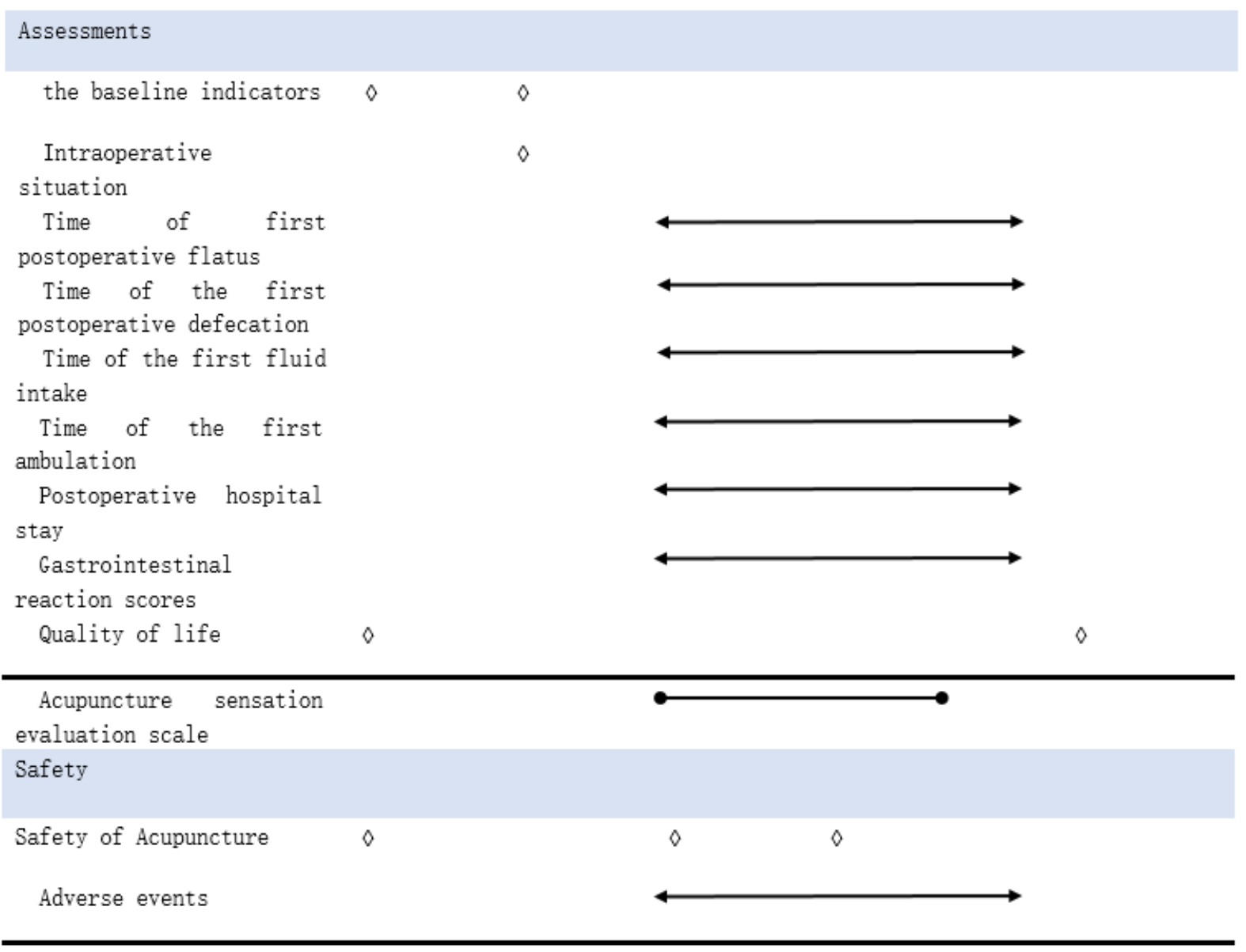

\section{Figure 2}

Time schedule of enrollment, interventions, and assessments 


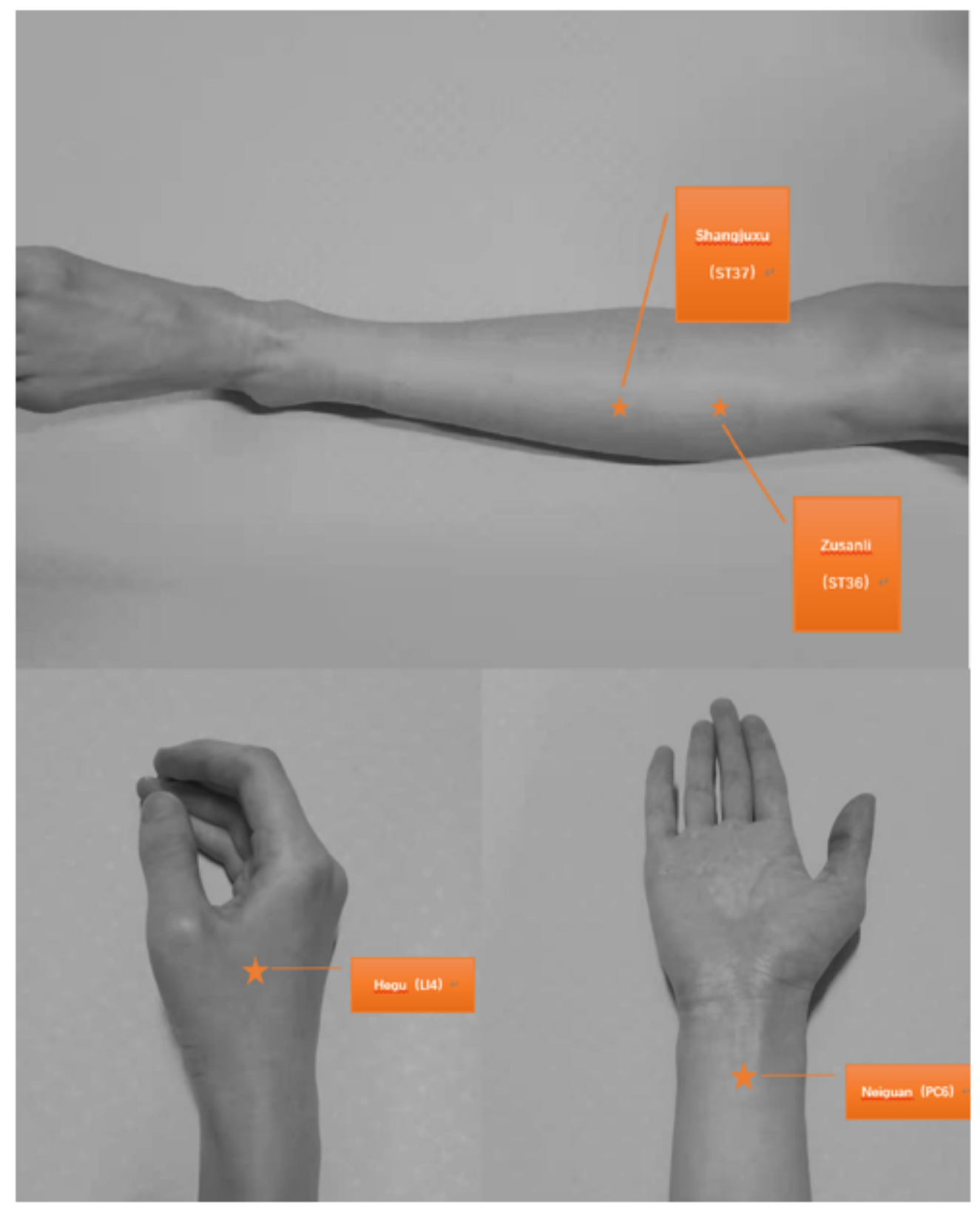

\section{Figure 3}

The location of ST36, ST37, L14, PC6. ST36 is the Zusanli acupoint; ST37 is the Shangjvxu acupoint; L14 is the Hegu acupoint; PC6 is the Neiguan acupoint 


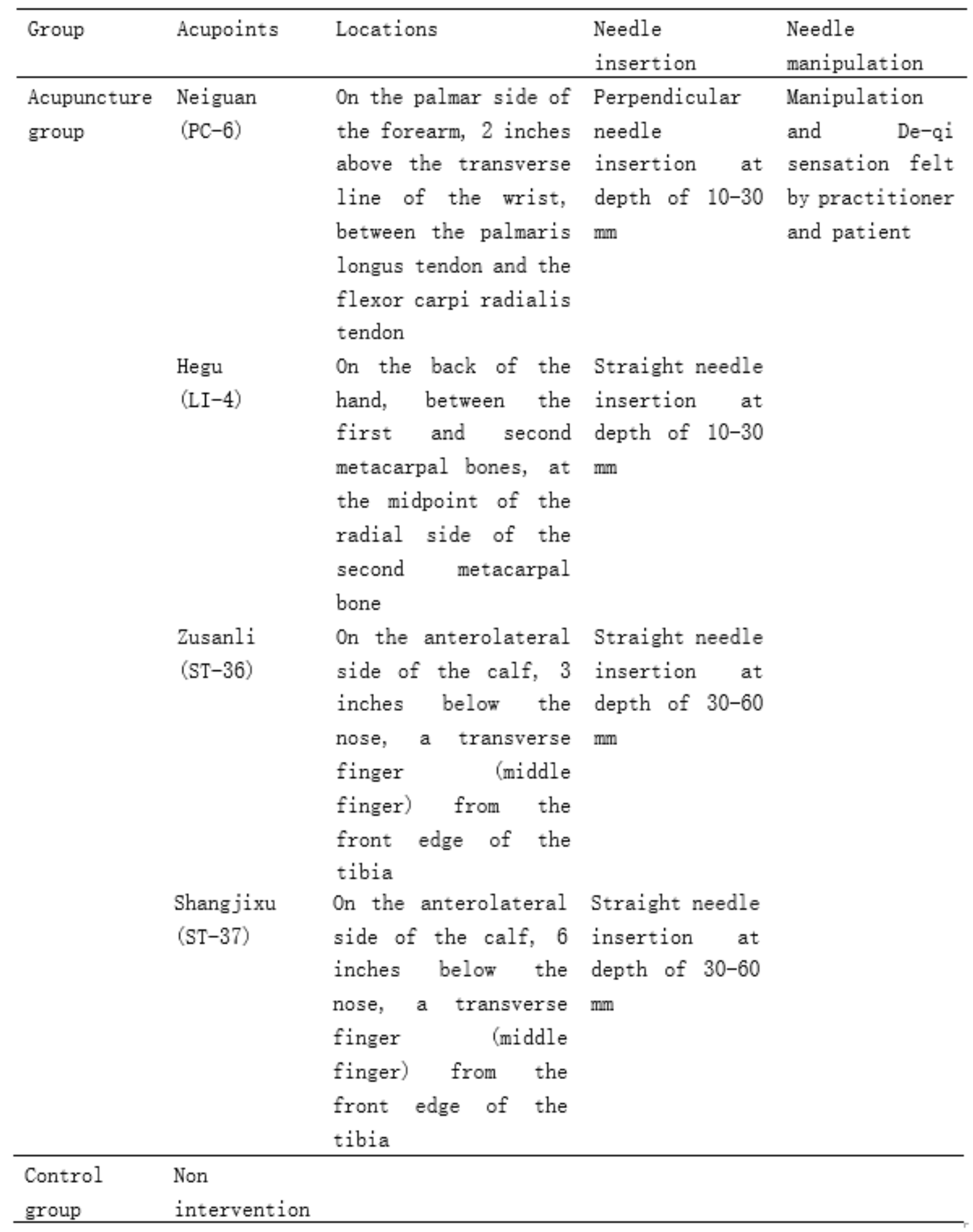

\section{Figure 4}

Locations and manipulations of acupuncture group and control group

\section{Supplementary Files}

This is a list of supplementary files associated with this preprint. Click to download. 
- renamedc3961.docx

- renamed4fdcd.docx

- renamed6a7b3.docx 